\title{
Strong LHCb evidence supporting the existence of the hidden-charm molecular pentaquarks
}

\author{
Rui Chen, ${ }^{1,2,3}$ Zhi-Feng Sun, ${ }^{1,2}$ Xiang Liu, ${ }^{1,2, *}$ and Shi-Lin $\mathrm{Zhu}^{3,4,5, \dagger}$ \\ ${ }^{1}$ School of Physical Science and Technology, Lanzhou University, Lanzhou 730000, China \\ ${ }^{2}$ Research Center for Hadron and CSR Physics, Lanzhou University \\ and Institute of Modern Physics of CAS, Lanzhou 730000, China \\ ${ }^{3}$ School of Physics and State Key Laboratory of Nuclear Physics and Technology, \\ Peking University, Beijing 100871, China \\ ${ }^{4}$ Collaborative Innovation Center of Quantum Matter, Beijing 100871, China \\ ${ }^{5}$ Center of High Energy Physics, Peking University, Beijing 100871, China
}

(Received 2 April 2019; published 10 July 2019)

\begin{abstract}
On 26 March 2019, at the Rencontres de Moriond QCD conference, the LHCb Collaboration reported the observation of three new pentaquarks, namely $P_{c}(4312), P_{c}(4440)$, and $P_{c}(4457)$, which are consistent with the loosely bound molecular hidden-charm pentaquark states composed of an $S$-wave charmed baryon $\Sigma_{c}$ and an $S$-wave anticharmed meson $\left(\bar{D}, \bar{D}^{*}\right)$. In this work, we present a direct calculation by the oneboson-exchange model and demonstrate explicitly that the $P_{c}(4312), P_{c}(4440)$, and $P_{c}(4457)$ do correspond to the loosely bound $\Sigma_{c} \bar{D}$ with $\left(I=1 / 2, J^{P}=1 / 2^{-}\right), \Sigma_{c} \bar{D}^{*}$ with $\left(I=1 / 2, J^{P}=1 / 2^{-}\right)$, and $\Sigma_{c} \bar{D}^{*}$ with $\left(I=1 / 2, J^{P}=3 / 2^{-}\right)$, respectively.
\end{abstract}

DOI: $10.1103 /$ PhysRevD.100.011502

\section{INTRODUCTION}

Finding the multiquark matter is an extremely important issue of hadron physics, which is full of challenge and opportunity not only for theorists but also for experimentalists. In the past 16 years, a series of observations of the charmoniumlike $X Y Z$ states and two $P_{c}$ states have inspired extensive investigations of the hidden-charm tetraquarks and pentaquarks, which enlarge our knowledge of the multiquark matter (see review articles [1-3] for more details).

Focusing on hidden-charm pentaquark, we must mention the predictions for the existence of the molecular type pentaquark [4-8] before LHCb's observation [9]. In 2015, $\mathrm{LHCb}$ announced the observation of two hidden-charm pentaquarks $P_{c}(4380)$ and $P_{c}(4450)$ in the $J / \psi p$ invariant mass spectrum of $\Lambda_{b} \rightarrow J / \psi K p$ [9]. This observation had stimulated extensive discussions on decoding their inner structures based on different configurations, which include the loosely bound molecular baryon-meson state [10-12] and tightly bound pentaquark state [13-16]. The experimental data in 2015 were unable to distinguish them.

\footnotetext{
*xiangliu@1zu.edu.cn

‘zhusl@pku.edu.cn
}

Published by the American Physical Society under the terms of the Creative Commons Attribution 4.0 International license. Further distribution of this work must maintain attribution to the author(s) and the published article's title, journal citation, and DOI. Funded by SCOAP .
Exploring the novel multiquark matter continues. Very recently, the LHCb Collaboration once again brought us a surprise. On 26 March 2019, at the Rencontres de Moriond QCD conference, the LHCb Collaboration reported the observation of three new pentaquarks [17]. By analyzing the $J / \psi p$ invariant mass spectrum, a new pentaquark named as the $P_{c}(4312)$ was discovered with $7.3 \sigma$ significance. And the $\mathrm{LHCb}$ updated analysis further indicates that the $P_{c}(4450)$ reported previously by $\mathrm{LHCb}$ [9] contains two narrow subpeaks, $P_{c}(4440)$ and $P_{c}(4457)$, which have $5.4 \sigma$ significance. Their resonance parameters include [18]

$$
\begin{aligned}
& P_{c}(4312)^{+}:\left\{\begin{array}{l}
m=4311.9 \pm 0.7_{-0.6}^{+6.8} \mathrm{MeV} \\
\Gamma=9.8 \pm 2.7_{-4.5}^{+3.7} \mathrm{MeV}
\end{array},\right. \\
& P_{c}(4440)^{+}:\left\{\begin{array}{l}
m=4440.3 \pm 1.3_{-4.7}^{+4.1} \mathrm{MeV} \\
\Gamma=20.6 \pm 4.9_{-10.1}^{+8.7} \mathrm{MeV}
\end{array}\right. \text {, } \\
& P_{c}(4457)^{+}:\left\{\begin{array}{l}
m=4457.3 \pm 1.3_{-4.1}^{+0.6} \mathrm{MeV} \\
\Gamma=6.4 \pm 2.0_{-1.9}^{+5.7} \mathrm{MeV}
\end{array} .\right.
\end{aligned}
$$

Since these three pentaquarks are observed in the $J / \psi p$ invariant mass spectrum, they have definite isospin quantum number $I=1 / 2$. We notice that the $P_{c}(4312)^{+}$is just below the $\Sigma_{c}^{+} \bar{D}^{0}$ threshold, while the masses of the $P_{c}(4440)^{+}$and $P_{c}(4457)^{+}$are slightly lower than the $\Sigma_{c}^{+} \bar{D}^{* 0}$ threshold (see Fig. 1).

By borrowing the experience of studying nuclear force, the one-boson-exchange (OBE) model was developed to 


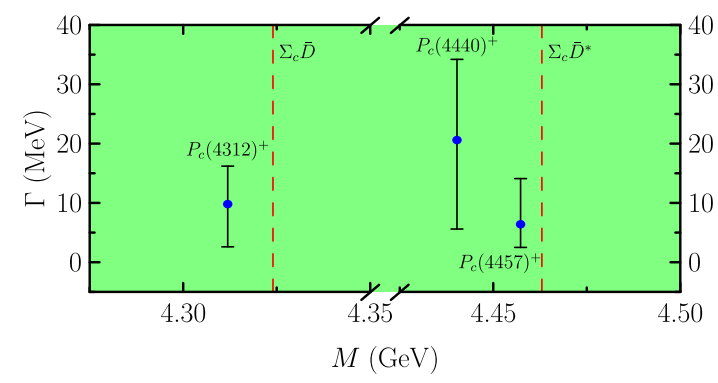

FIG. 1. The observed three pentaquarks from $\mathrm{LHCb}$ and comparison with the thresholds.

investigate these observed charmoniumlike $X Y Z$ states [19-24]. Later, in 2011, we applied the OBE model to study the $\Sigma_{c} \bar{D}$ and $\Sigma_{c} \bar{D}^{*}$ interactions [5]. Although the treatment of the $\Sigma_{c} \bar{D}$ and $\Sigma_{c} \bar{D}^{*}$ systems in Ref. [5] was quite simple and straightforward, the existence of the $\Sigma_{c} \bar{D}$ and $\Sigma_{c} \bar{D}^{*}$ molecular hidden-charm pentaquark was predicted clearly, which has become one of the pioneering papers exploring the molecular hidden-charm pentaquarks.

Until now, the OBE model has been greatly improved compared with the situation in 2011 (see review [1]), which stimulates us to include the coupled-channel effect and $S-D$ wave mixing in the calculation. Especially, the new observations of three pentaquarks announced by $\mathrm{LHCb}$ makes the study of the hidden-charm molecular pentaquarks enter upon a new era with higher precision.

In this paper, we will present a precise investigation of the $\Sigma_{c} \bar{D}$ and $\Sigma_{c} \bar{D}^{*}$ systems, from which the readers can learn why this baryon-meson configuration of the hiddencharm pentaquark [5] is connected with the LHCb new measurement [17]. For the $\Sigma_{c} \bar{D}$ and $\Sigma_{c} \bar{D}^{*}$ systems, there are only three molecular states with isospin $I=1 / 2$. The newly observed $P_{c}(4312)^{+}$corresponds to the $\Sigma_{c} \bar{D}$ with $I\left(J^{P}\right)=1 / 2\left(1 / 2^{-}\right)$, while the $P_{c}(4440)^{+}$and $P_{c}(4457)^{+}$ can be identified as the $\Sigma_{c} \bar{D}$ molecular states with $I\left(J^{P}\right)=$ $1 / 2\left(1 / 2^{-}\right)$and $1 / 2\left(3 / 2^{-}\right)$, respectively. The updated LHCb measurement [17] confirms the existence of molecular hidden-charm pentaquarks composed of an $S$-wave charmed baryon and an $S$-wave anticharmed meson [4-8,10-12], which shall become a milestone in the exploration of the multiquark hadronic matter.

\section{REPRODUCING THREE PENTAQUARKS}

In the framework of the OBE model, a key point is the deduction of the effective potentials for the hadronic molecular systems. Considering the coupled channel effect and $S$ - $D$ wave mixing, we may list the corresponding channel contributions to the $\Sigma_{c} \bar{D}$ system with $J^{P}=1 / 2^{-}$, two $\Sigma_{c} \bar{D}^{*}$ systems with $J^{P}=1 / 2^{-}, 3 / 2^{-}$, and the $\Sigma_{c}^{*} \bar{D}$ system with $J^{P}=3 / 2^{-}$, which can be categorized into two groups

$$
\begin{aligned}
1 / 2^{-}: & \left.\left.\left.\Sigma_{c} \bar{D}\right|^{2} \mathbb{S}_{\frac{1}{2}}\right\rangle,\left.\Sigma_{c}^{*} \bar{D}\right|^{4} \mathbb{D}_{\frac{1}{2}}\right\rangle, \Sigma_{c} \bar{D}^{*}\left|{ }^{2} \mathbb{S}_{\frac{1}{2}} / 4 \mathbb{D}_{\frac{1}{2}}\right\rangle, \\
& \Sigma_{c}^{*} \bar{D}^{*}\left|{ }^{2} \mathbb{S}_{\frac{1}{2}} /{ }^{4} \mathbb{D}_{\frac{1}{2}} /{ }^{6} \mathbb{D}_{\frac{1}{2}}\right\rangle, \\
3 / 2^{-}: & \left.\left.\Sigma_{c} \bar{D}^{*}\right|^{4} \mathbb{S}_{\frac{3}{2}} /{ }^{2} \mathbb{D}_{\frac{3}{2}} /{ }^{4} \mathbb{D}_{\frac{3}{2}}\right\rangle, \Sigma_{c}^{*} \bar{D}^{*}\left|{ }^{4} \mathbb{S}_{\frac{3}{2}} /{ }^{2} \mathbb{D}_{\frac{3}{2}} /{ }^{4} \mathbb{D}_{\frac{3}{2}} /{ }^{6} \mathbb{D}_{\frac{3}{2}}\right\rangle
\end{aligned}
$$

The involved flavor wave functions $\left|I, I_{3}\right\rangle$ are

$$
\begin{aligned}
\left|\frac{1}{2}, \frac{1}{2}\right\rangle & =\sqrt{\frac{2}{3}}\left|\Sigma_{c}^{(*)++} D^{(*)-}\right\rangle-\frac{1}{\sqrt{3}}\left|\Sigma_{c}^{(*)+} \bar{D}^{(*) 0}\right\rangle \\
\left|\frac{1}{2},-\frac{1}{2}\right\rangle & =\frac{1}{\sqrt{3}}\left|\Sigma_{c}^{(*)+} D^{(*)-}\right\rangle-\sqrt{\frac{2}{3}}\left|\Sigma_{c}^{(*) 0} \bar{D}^{(*) 0}\right\rangle .
\end{aligned}
$$

In order to calculate the effective potentials of the hiddencharm molecular baryons, we need to utilize the effective Lagrangian describing the interactions of the charmed or anticharmed baryon/meson with the light mesons. The Lagrangian depicting the interaction of the ground state charmed baryons with the light mesons can be constructed according to the chiral symmetry, the heavy quark symmetry, and the hidden local symmetry [25], while the one for the $S$-wave pseudoscalar and vector charmed mesons according to the chiral symmetry and the heavy quark symmetry [26-30]. Here, we list the Lagrangians in the following:

$$
\begin{aligned}
\mathcal{L}_{H}= & g_{S}\left\langle\bar{H}_{a} \sigma H_{b}\right\rangle+i g\left\langle\bar{H}_{a} \gamma_{\mu} A_{a b}^{\mu} \gamma_{5} H_{b}\right\rangle \\
& -i \beta\left\langle\bar{H}_{a} v_{\mu}\left(\mathcal{V}_{a b}^{\mu}-\rho_{a b}^{\mu}\right) H_{b}\right\rangle+i \lambda\left\langle\bar{H}_{a} \sigma_{\mu \nu} F^{\mu \nu}(\rho) H_{b}\right\rangle,
\end{aligned}
$$

$$
\begin{aligned}
\mathcal{L}_{\mathcal{S}}= & \left.l_{S}\left\langle\overline{\mathcal{S}}_{\mu} \sigma \mathcal{S}^{\mu}\right\rangle-\frac{3}{2} g_{1} \varepsilon^{\mu \nu \lambda \kappa} v_{\kappa}\left\langle\overline{\mathcal{S}}_{\mu} A_{\nu} \mathcal{S}_{\lambda}\right\rangle\right\rangle \\
& +i \beta_{S}\left\langle\overline{\mathcal{S}}_{\mu} v_{\alpha}\left(\mathcal{V}_{a b}^{\alpha}-\rho_{a b}^{\alpha}\right) \mathcal{S}^{\mu}\right\rangle+\lambda_{S}\left\langle\overline{\mathcal{S}}_{\mu} F^{\mu \nu}(\rho) \mathcal{S}_{\nu}\right\rangle
\end{aligned}
$$

Here, the following definitions are used, i.e., $H=\left[\tilde{\mathcal{P}}^{* \mu} \gamma_{\mu}-\right.$ $\left.\tilde{\mathcal{P}} \gamma_{5}\right] \frac{1-\gamma}{2}, \quad \mathcal{S}_{\mu}=-\sqrt{\frac{1}{3}}\left(\gamma_{\mu}+v_{\mu}\right) \gamma^{5} \mathcal{B}_{6}+\mathcal{B}_{6 \mu}^{*}, \quad \bar{H}=\gamma_{0} H^{\dagger} \gamma_{0}$, $\overline{\mathcal{S}}_{\mu}=\mathcal{S}_{\mu}^{\dagger} \gamma_{0}, A_{\mu}=\frac{1}{2}\left(\xi^{\dagger} \partial_{\mu} \xi-\xi \partial_{\mu} \xi^{\dagger}\right)=\frac{i}{f_{\pi}} \partial_{\mu} \mathbb{P}+\cdots, \mathcal{V}_{\mu}=$ $\frac{1}{2}\left(\xi^{\dagger} \partial_{\mu} \xi-\xi \partial_{\mu} \xi^{\dagger}\right)=\frac{i}{2 f_{\pi}^{2}}\left[\mathbb{P}, \partial_{\mu} \mathbb{P}\right]+\cdots, \quad \xi=\exp \left(i \mathbb{P} / f_{\pi}\right)$, $\rho^{\mu}=i g_{V} \mathbb{V}^{\mu} / \sqrt{2}$, and $F^{\mu \nu}(\rho)=\partial^{\mu} \rho^{\nu}-\partial^{\nu} \rho^{\mu}+\left[\rho^{\mu}, \rho^{\nu}\right]$. And $\tilde{\mathcal{P}}$ and $\tilde{\mathcal{P}}^{*}$ satisfy $\tilde{\mathcal{P}}=\left(\bar{D}^{0}, D^{-}\right)^{T}, \tilde{\mathcal{P}}^{*}=\left(\bar{D}^{* 0}, D^{*-}\right)^{T}$. Note that the $\mathrm{SU}(3)$ chiral symmetry is considered to construct the Lagrangians. However, for convenience, we only show the first two lines and columns in the above matrices, since we do not consider, in this paper, the strange case which corresponds to the third line and column. Thus, the $\mathcal{B}_{3}, \mathcal{B}_{6}$, and $\mathcal{B}_{6}^{*}$ are defined as 


$$
\begin{aligned}
& \mathbb{P}=\left(\begin{array}{cc}
\frac{\pi^{0}}{\sqrt{2}}+\frac{\eta}{\sqrt{6}} & \pi^{+} \\
\pi^{-} & -\frac{\pi^{0}}{\sqrt{2}}+\frac{\eta}{\sqrt{6}}
\end{array}\right), \quad \mathcal{B}_{6}^{(*)}=\left(\begin{array}{cc}
\Sigma_{c}^{(*)++} & \frac{\Sigma_{c}^{(*)+}}{\sqrt{2}} \\
\frac{\Sigma_{c}^{(*)+}}{\sqrt{2}} & \Sigma_{c}^{(*) 0}
\end{array}\right), \\
& \mathbb{V}=\left(\begin{array}{cc}
\frac{\rho^{0}}{\sqrt{2}}+\frac{\omega}{\sqrt{2}} & \rho^{+} \\
\rho^{-} & -\frac{\rho^{0}}{\sqrt{2}}+\frac{\omega}{\sqrt{2}}
\end{array}\right) .
\end{aligned}
$$

With the above preparation, we consider the $t$-channel Feynman diagrams to deduce the effective potentials, where the Breit approximation $\mathcal{V}_{E}(\boldsymbol{q})=$ $-\mathcal{M} / \sqrt{\prod_{i} 2 M_{i} \prod_{f} 2 M_{f}}$ is needed to relate the $t$-channel scattering amplitudes $\mathcal{M}$ to the potentials $\mathcal{V}_{E}(\boldsymbol{q})$ in the momentum space. The Fourier transformation is performed to obtain the potentials in the coordinate space. Besides, the monopole form factor $\mathcal{F}\left(q^{2}, m_{E}^{2}\right)=\left(\Lambda^{2}-m_{E}^{2}\right) /\left(\Lambda^{2}-q^{2}\right)$ is introduced for compensating the off shell effect of the exchanged meson and describing the structure effect of every interaction vertex. According to the experience of the deuteron and interaction between the proton and neutron, cutoff $\Lambda$ in the form factor is taken around $1 \mathrm{GeV}$, which is widely used to test whether a loose molecular state exists or not.

In the OBE model, the general expressions of the potentials in the coordinate space for the discussed systems include scalar, pseudoscalar, and vector mesons exchanges part, i.e.,

$$
V^{i j}(r)=V_{\pi / \eta}^{i j}(r)+V_{\rho / \omega}^{i j}(r)+V_{\sigma}^{i j}(r),
$$

according to the mass difference of exchanged mesons, $\pi$, $\sigma / \eta$, and $\rho / \omega$ exchanges contribute in long, intermediate, and short range. For $J=1 / 2, i, j=1,2,3,4$ corresponds to the channels $\Sigma_{c} \bar{D}, \Sigma_{c}^{*} \bar{D}, \Sigma_{c} \bar{D}^{*}, \Sigma_{c}^{*} \bar{D}^{*}$, while for $J=3 / 2$, $i, j=1,2$ corresponds to $\Sigma_{c} \bar{D}^{*}, \Sigma_{c}^{*} \bar{D}^{*}$. For convenience, we define the following expressions:

$$
\begin{aligned}
& Y(\Lambda, m, r)= \frac{1}{4 \pi r}\left(e^{-m r}-e^{-\Lambda r}\right)-\frac{\Lambda^{2}-m^{2}}{8 \pi \Lambda} e^{-\Lambda r}, \\
& \mathcal{Y}_{\Lambda m_{a}}^{i j}=\mathcal{D}_{i j} Y\left(\Lambda, m_{\sigma}, r\right), \\
& \mathcal{Z}_{\Lambda m_{a}}^{i j}=\left(\mathcal{E}_{i j} \nabla^{2}+\mathcal{F}_{i j} r \frac{\partial}{\partial r} \frac{1}{r} \frac{\partial}{\partial r}\right) Y\left(\Lambda, m_{a}, r\right), \\
& \mathcal{Z}_{\Lambda m_{a}}^{\prime i j}=\left(2 \mathcal{E}_{i j} \nabla^{2}-\mathcal{F}_{i j} r \frac{\partial}{\partial r} \frac{1}{r} \frac{\partial}{\partial r}\right) Y\left(\Lambda, m_{a}, r\right),
\end{aligned}
$$

where the matrices $\mathcal{D}_{i j}, \mathcal{E}_{i j}$ correspond to the spin-spin operators and $\mathcal{F}_{i j}$ to the tensor operator, e.g., for the process $\Sigma_{c} \bar{D}^{*} \rightarrow \Sigma_{c} \bar{D}^{*}$,

$$
\mathcal{D}=\boldsymbol{\epsilon}_{2} \cdot \boldsymbol{\epsilon}_{4}^{\dagger}, \quad \mathcal{E}=\boldsymbol{\sigma} \cdot\left(i \boldsymbol{\epsilon}_{2} \times \boldsymbol{\epsilon}_{4}^{\dagger}\right), \quad \mathcal{F}=S\left(\hat{r}, \boldsymbol{\sigma}, i \boldsymbol{\epsilon}_{2} \times \boldsymbol{\epsilon}_{4}^{\dagger}\right) .
$$

In a numerical calculation, these operators should be sandwiched between the discussed spin-orbit wave functions, all of the operators will be replaced by serials of nonzero matrix elements, for example, for $\Sigma_{c} \bar{D}^{*} \rightarrow \Sigma_{c} \bar{D}^{*}$ with $J^{P}=1 / 2^{-}, \quad\left\langle\Sigma_{c} \bar{D}^{*}\left(1 / 2^{-}\right)|\mathcal{E}| \Sigma_{c} \bar{D}^{*}\left(1 / 2^{-}\right)\right\rangle=\left(\begin{array}{cc}-2 & 0 \\ 0 & 1\end{array}\right)$. The subpotentials now can be written as

$$
\begin{aligned}
& V^{11}=-A Y\left(\Lambda, m_{\sigma}, r\right)-\frac{\mathcal{G} B}{2} Y\left(\Lambda, m_{\rho}, r\right)-\frac{B}{4} Y\left(\Lambda, m_{\omega}, r\right), \\
& V^{12}=\frac{A}{\sqrt{3}} \mathcal{Y}_{\Lambda_{3} m_{\sigma 3}}+\frac{\mathcal{G} B}{2 \sqrt{3}} \mathcal{Y}_{\Lambda_{3}, m_{\rho 3}}^{12}+\frac{B}{4 \sqrt{3}} \mathcal{Y}_{\Lambda_{3}, m_{\omega 3}}^{12}, \\
& V^{13}=\frac{\mathcal{G} C}{3} \mathcal{Z}_{\Lambda_{4}, m_{\pi 4}}^{13}+\frac{C}{18} \mathcal{Z}_{\Lambda_{4}, m_{\eta 4}}^{13}+\frac{2 \mathcal{G} D}{9} \mathcal{Z}_{\Lambda_{4}, m_{\rho 4}}^{\prime 13}+\frac{D}{9} \mathcal{Z}_{\Lambda_{4}, m_{\omega 4}}^{\prime 13}, \\
& V^{14}=\frac{\mathcal{G} C}{2 \sqrt{3}} \mathcal{Z}_{\Lambda_{5}, m_{\pi 5}}^{14}+\frac{C}{12 \sqrt{3}} \mathcal{Z}_{\Lambda_{5}, m_{\eta 5}}^{14} \\
& +\frac{\mathcal{G} D}{3 \sqrt{3}} \mathcal{Z}_{\Lambda_{5}, m_{\rho 5}}^{\prime 14}+\frac{D}{6 \sqrt{3}} \mathcal{Z}_{\Lambda_{5}, m_{\omega 5}}^{\prime 14}, \\
& V^{22}=-A \mathcal{Y}_{\Lambda, m_{\sigma}}^{11}-\frac{\mathcal{G} B}{2} \mathcal{Y}_{\Lambda, m_{\rho}}^{22}-\frac{B}{4} \mathcal{Y}_{\Lambda, m_{\omega}}^{22} \\
& V^{23}=\frac{\mathcal{G} C}{2 \sqrt{3}} \mathcal{Z}_{\Lambda_{0}, m_{\pi 0}}^{23}+\frac{C}{12 \sqrt{3}} \mathcal{Z}_{\Lambda_{0}, m_{\eta 0}}^{23}-\frac{\mathcal{G} D}{3 \sqrt{3}} \mathcal{Z}_{\Lambda_{0}, m_{\rho 0}}^{123} \\
& -\frac{D}{6 \sqrt{3}} \mathcal{Z}_{\Lambda_{0}, m_{\omega 0}}^{\prime 23} \\
& V^{24}=\frac{\mathcal{G} C}{2} \mathcal{Z}_{\Lambda_{1}, m_{\pi 1}}^{24}+\frac{C}{12} \mathcal{Z}_{\Lambda_{1}, m_{\eta 1}}^{24}-\frac{\mathcal{G} D}{3} \mathcal{Z}_{\Lambda_{1}, m_{\rho 1}}^{24}-\frac{D}{6} \mathcal{Z}_{\Lambda_{1}, m_{\omega 1}}^{24}, \\
& V^{33}=-A \mathcal{Y}_{\Lambda, m_{\sigma}}^{33}+\frac{\mathcal{G} C}{3} \mathcal{Z}_{\Lambda, m_{\pi}}^{33}+\frac{C}{18} \mathcal{Z}_{\Lambda, m_{\eta}}^{33}-\frac{\mathcal{G} B}{2} \mathcal{Y}_{\Lambda, m_{\rho}}^{33} \\
& -\frac{2 \mathcal{G} D}{9} \mathcal{Z}_{\Lambda, m_{\rho}}^{133}-\frac{B}{4} \mathcal{Y}_{\Lambda, m_{\omega}}^{33}-\frac{D}{9} \mathcal{Z}_{\Lambda, m_{\omega}}^{133}, \\
& V^{34}=\frac{A}{\sqrt{3}} \mathcal{Y}_{\Lambda_{2}, m_{\sigma 2}}^{34}+\frac{\sqrt{3} \mathcal{G} C}{6} \mathcal{Z}_{\Lambda_{2}, m_{\pi 2}}^{34}+\frac{\sqrt{3} C}{36} \mathcal{Z}_{\Lambda_{2}, m_{\eta 2}}^{34} \\
& +\frac{\mathcal{G} B}{2 \sqrt{3}} \mathcal{Y}_{\Lambda_{2}, m_{\rho 2}}^{34}-\frac{\mathcal{G} D}{3 \sqrt{3}} \mathcal{Z}_{\Lambda_{2}, m_{\rho 2}}^{134}+\frac{B}{4 \sqrt{3}} \mathcal{Y}_{\Lambda_{2}, m_{\omega 2}}^{34} \\
& -\frac{D}{6 \sqrt{3}} \mathcal{Z}_{\Lambda_{2}, m_{\omega 2}}^{\prime 34} \\
& V^{44}=-A \mathcal{Y}_{\Lambda, m_{\sigma}}^{44}-\frac{\mathcal{G} C}{2} \mathcal{Z}_{\Lambda, m_{\pi}}^{44}-\frac{C}{12} \mathcal{Z}_{\Lambda, m_{\eta}}^{44}-\frac{\mathcal{G B}}{2} \mathcal{Y}_{\Lambda, m_{\rho}}^{44} \\
& +\frac{\mathcal{G} D}{3} \mathcal{Z}_{\Lambda, m_{\rho}}^{\prime 44}-\frac{B}{4} \mathcal{Y}_{\Lambda, m_{\omega}}^{44}+\frac{D}{6} \mathcal{Z}_{\Lambda, m_{\omega}}^{\prime 44} .
\end{aligned}
$$

Here, $A=l_{S} g_{S}, B=\beta \beta_{S} g_{V}^{2}, C=\frac{g g_{1}}{f_{\pi}^{2}}, D=\lambda \lambda_{S} g_{V}^{2}, \mathcal{G}$ is the isospin factor, which is taken as -1 for the isospin $1 / 2$ system (for the isospin $3 / 2$ system $\mathcal{G}=1 / 2$ ). The variables in these functions are defined as $\Lambda_{b}^{2}=\Lambda^{2}-q_{b}^{2}, m_{b}^{2}=m^{2}-q_{b}^{2}$, with $b=0,1, \ldots, 5$, and $q_{i}^{2}$ s are the functions of the masses of the charmed mesons and baryons whose values are $q_{0}=35.60 \mathrm{MeV}, q_{1}=61.41 \mathrm{MeV}, q_{2}=25.08 \mathrm{MeV}$, $q_{3}=97.38 \mathrm{MeV}, q_{4}=60.53 \mathrm{MeV}, q_{5}=35.45 \mathrm{MeV}$.

The values of the coupling constants are taken from $[19,25,29,30], \quad g_{S}=0.76, \quad g=0.59, \quad \beta=0.9, \quad l_{S}=6.2$, 

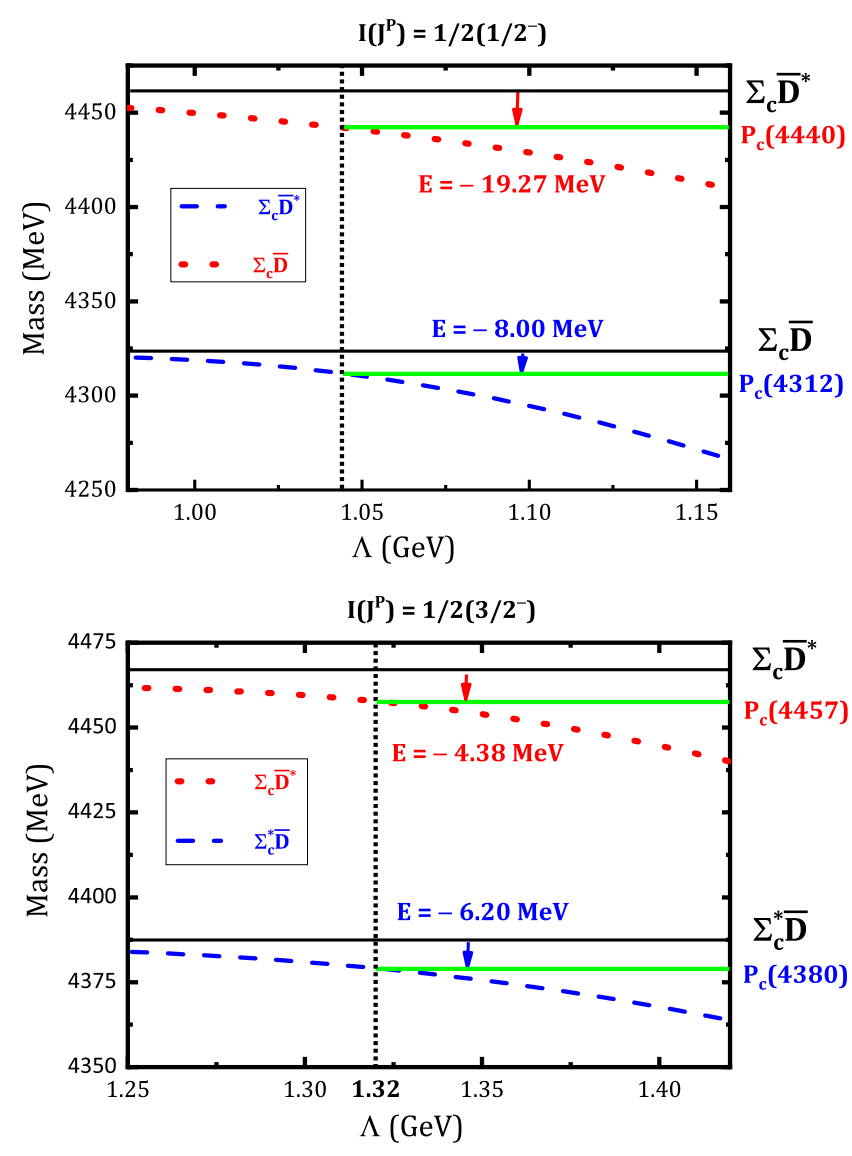

FIG. 2. The $\Lambda$ dependence of the bound state masses for the coupled channel $\Sigma_{c}^{(*)} \bar{D}^{(*)}$ systems with $I=1 / 2$. Here, $E$ is the binding energy. The vertical dotted lines and horizontal solid lines stand for the cutoff $\Lambda$ value and hidden-charm mesonbaryon systems thresholds.

$g_{1}=0.94, \beta_{S}=-1.74, \lambda=0.56 \mathrm{GeV}^{-1}, \lambda_{S}=-3.31 \mathrm{GeV}^{-1}$, and $g_{V}=5.9$. Here, the coupling constants for the pion exchange $g$ and $g_{1}$ are determined from the experiment data, $\Gamma\left(D^{*} \rightarrow D \pi\right)$ and $\Gamma\left(\Sigma_{c}^{(*)} \rightarrow \Lambda_{c} \pi\right)$ [31]. For the scalar and vector mesons exchanges, the coupling constants are estimated in the quark model, where the average of the coupling vertex is simultaneously calculated, at hadron level and at quark level, and the heavy quark does not couple to the light mesons. The signs are fixed with the help of the quark model.

Finally, the binding energies for the $\Sigma_{c} \bar{D}$ and $\Sigma_{c} \bar{D}^{*}$ systems can be evaluated by solving the Schrödinger equation. In Fig. 2, we present the bound state masses for the coupled channel $\Sigma_{c}^{(*)} \bar{D}^{(*)}$ systems with $I=1 / 2$ which depends on the cutoff $\Lambda$. As the cutoff increases, the binding energy of all the systems becomes deeper and deeper.

For the $J^{P}=1 / 2^{-}$case, we can reproduce the masses of the $P_{c}(4312)$ and $P_{c}(4440)$ with a very reasonable cutoff value $\Lambda=1.04 \mathrm{GeV}$. The binding energies for these systems are $-8.00 \mathrm{MeV}$ and $-19.27 \mathrm{MeV}$, respectively. Their corresponding root-mean-square (RMS) radii are
1.22 and $0.88 \mathrm{fm}$, respectively. For the $P_{c}(4312)$, the $\Sigma_{c} \bar{D}$ channel is dominant, the probabilities of the $\Sigma_{c} \bar{D}$, $\Sigma_{c} \bar{D}^{*}$, and $\Sigma_{c}^{*} \bar{D}^{*}$ components are around $84 \%, 12 \%$, and $4 \%$, respectively. The $P_{c}(4440)$ is mainly composed of the $S$-wave $\Sigma_{c} \bar{D}^{*}$ component with the probability over $94 \%$.

For the $I\left(J^{P}\right)=1 / 2\left(3 / 2^{-}\right)$case, as shown in Fig. 2, the mass of the $P_{c}(4457)$ is also reproduced with $\Lambda=1.32 \mathrm{GeV}$. Here, its binding energy and RMS radius are $-4.38 \mathrm{MeV}$ and $1.61 \mathrm{fm}$, respectively. The coupled-channel effect plays an important role, since the probabilities of the $\Sigma_{c} \bar{D}^{*}$ and $\Sigma_{c}^{*} \bar{D}^{*}$ components are around $75 \%$ and $25 \%$, respectively.

With the same value of the cutoff as that of $P_{c}(4457)$, we find another bound state of the quantum number $I\left(J^{P}\right)=1 / 2\left(3 / 2^{-}\right)$, which locates below the $\Sigma_{c}^{*} \bar{D}$ threshold with a binding energy $E=-6.20 \mathrm{MeV}$ and RMS radius $r_{\mathrm{RMS}}=1.40 \mathrm{fm}$. The dominant channel is the $\Sigma_{c}^{*} \bar{D}\left({ }^{4} S_{3 / 2}\right)$ with a probability over $87 \%$. This state can be identified with the $P_{c}^{+}(4380)$ observed in 2015 also by LHCb Collaboration [9], although the recent $\mathrm{LHCb}$ measurement does not give any information of such a particle. We hope the further experiments can provide a more precise measurement and confirm the existence of this state.

From the above discussion, we see that the three resonances $P_{c}^{+}(4312), P_{c}^{+}(4440), P_{c}^{+}$(4457) are naturally interpreted within the loosely bound $\Sigma_{c} \bar{D}$ and $\Sigma_{c} \bar{D}^{*}$ molecule picture. For the $\Sigma_{c} \bar{D}^{(*)}$ systems, there are only three molecular states with isospin $1 / 2$, i.e., $\Sigma_{c} \bar{D}$ corresponding to the lower peak $P_{c}(4312)^{+}$, and $\Sigma_{c} \bar{D}^{*}$ to the higher peaks $P_{c}(4440)$ and $P_{c}(4457)$. Within the hiddencharm molecular assignment, we can easily understand why the two higher peaks are very close to each other, and the mass difference between the two higher peaks and the lowest $P_{c}^{+}(4312)$ peak is around $140 \mathrm{MeV}$ which is approximately the mass difference of the $D$ and $D^{*}$ mesons.

\section{MORE PREDICTIONS}

Besides explaining the three particles $P_{c}(4312)$, $P_{c}(4440)$, and $P_{c}(4457)$, we further investigate the possible existence of the $\Sigma_{c}^{(*)} \bar{D}^{(*)}$ with $\left(I=\frac{3}{2}, J^{P}=\frac{1}{2}^{-} / \frac{3}{2}^{-}\right)$. In Table I, we collect the corresponding bound state solutions. However, when the bound state solutions emerge, the corresponding cutoffs are around $2-4 \mathrm{GeV}$ and quite large for all these systems. The past experience indicates that such a large cutoff value sometimes points to the nonexistence of the molecular states. Consequently, we cannot give a clear conclusion whether the $\Sigma_{c}^{(*)} \bar{D}^{(*)}$ systems with isospin $3 / 2$ exist or not.

In the experimental process $\Lambda_{b} \rightarrow J / \psi p K$, all the $P_{c}$ states are produced by the weak decay of $\Lambda_{b}^{0}$. With the assignment that the $P_{c}(4312)$ and $P_{c}(4440) / P_{c}(4457)$ are the $\Sigma_{c} \bar{D}$ and $\Sigma_{c} \bar{D}^{*}$ molecules, respectively, these states can transit to the final $J / \psi p$ by exchanging an $S$-wave charmed meson, and the $J / \psi$ and $p$ reside in an $S$ wave. 
TABLE I. Bound state solutions for the possible hidden-charm molecular pentaquarks $\Sigma_{c}^{(*)} \bar{D}^{(*)}$ with $I=3 / 2$. The units for cutoff $\Lambda, E$, and root-mean-square radius $r_{\mathrm{RMS}}$ are $\mathrm{GeV}, \mathrm{MeV}$, and $\mathrm{fm}$, respectively.

\begin{tabular}{lccccc}
\hline \hline$\Lambda$ & $E$ & $r_{\mathrm{RMS}}$ & $\Lambda$ & $E$ & $r_{\mathrm{RMS}}$ \\
\hline \multicolumn{3}{c}{$\Sigma_{c} \bar{D} J^{P}=1 / 2^{-}$} & & \multicolumn{3}{c}{$\Sigma_{c}^{*} \bar{D} J^{P}=3 / 2^{-}$} \\
\hline 2.26 & -0.22 & 4.98 & 3.04 & -1.85 & 0.80 \\
2.32 & -5.40 & 1.35 & 3.05 & -6.03 & 0.49 \\
2.38 & -16.17 & 0.82 & 3.06 & -10.43 & 0.42 \\
& $\Sigma_{c} \bar{D}^{*} J^{P}=1 / 2^{-}$ & & \multicolumn{3}{c}{$\Sigma_{c} \bar{D}^{*} J^{P}=3 / 2^{-}$} \\
4.21 & -0.63 & 2.57 & 2.97 & -0.41 & 4.18 \\
4.23 & -5.48 & 0.77 & 3.03 & -4.80 & 1.38 \\
4.25 & -11.33 & 0.58 & 3.09 & -13.46 & 0.86 \\
\hline \hline
\end{tabular}

The molecular systems $\Sigma_{c}^{(*)} \bar{D}^{(*)} \quad$ with $\quad\left(I=\frac{3}{2}\right.$, $\left.J^{P}=\frac{1}{2}^{-} / \frac{3}{2}^{-}\right)$, if they should exist, cannot decay into $J / \psi p$ due to the isospin conservation. A possible decay mode is $J / \psi \Delta(1232)$ for both $J=1 / 2$ and $3 / 2$, which will be helpful for future experimental search.

\section{SUMMARY}

Very recently, $\mathrm{LHCb}$ reported the observation of three new pentaquarks $P_{c}(4312)^{+}, P_{c}(4440)^{+}$, and $P_{c}(4457)^{+}$ in the $J / \psi p$ invariant mass spectrum of $\Lambda_{b} \rightarrow J / \psi p K$ [17], which benefitted from large data samples collected at LHC Run 1 and Run 2. This new discovery further opens the mysterious Pandora's box of exploring exotic multiquark states. This new $\mathrm{LHCb}$ observation for the first time confirmed the existence of the baryon-meson configuration molecular hidden-charm pentaquarks in experiment, which should become a milestone of the exploration of the multiquark hadronic matter. In this work, we have performed a phenomenological study to decode the inner structure of the newly observed $P_{c}(4312), P_{c}(4440)$, and $P_{c}(4457)$ based on the OBE model after the inclusion of the coupled-channel effect and $S$ - $D$ wave mixing. The consistence of the LHCb new observation with the hiddencharm molecular pentaquarks with the baryon-meson configuration is clearly demonstrated.

In this work, we have also studied the interaction of the $\Sigma_{c}^{*} \bar{D}$ system in the framework of the OBE model and found the existence of a $\Sigma_{c}^{*} \bar{D}$ molecular hidden-charm pentaquark with $I\left(J^{P}\right)=1 / 2\left(3 / 2^{-}\right)$, which has a mass $4379.11 \mathrm{MeV}$. This predicted $\Sigma_{c}^{*} \bar{D}$ molecular hidden-charm pentaquark may correspond to the $P_{c}(4380)$ reported by LHCb in 2015 [9]. However, the talk at the Rencontres de Moriond QCD conference [17] did not mention $P_{c}(4380)$. After checking the new experimental data released by LHCb [18], one may notice the evidence of the accumulation of events around $4380 \mathrm{MeV}$ in the $J / \psi p$ invariant mass spectrum. Moreover, we have also studied the possible existence of the hiddencharm molecular pentaquarks with isospin $I=3 / 2$, which are the isospin partners of the newly observed $P_{c}(4312)^{+}$, $P_{c}(4440)^{+}$, and $P_{c}(4457)^{+}$. These states should also be searched for at LHCb. The past 16 years has been the golden age of the search of the exotic hadrons.

\section{ACKNOWLEDGMENTS}

This project is supported by the National Natural Science Foundation of China under Grants No. 11705069 and No. 11575008, and National Key Basic Research Program of China (Grant No. 2015CB856700). R. C. is also supported by the National Postdoctoral Program for Innovative Talent. X. L. is also supported by the National Program for Support of Top-notch Young Professionals and the China National Funds for Distinguished Young Scientists under Grant No. 11825503.
[1] H. X. Chen, W. Chen, X. Liu, and S. L. Zhu, The hiddencharm pentaquark and tetraquark states, Phys. Rep. 639, 1 (2016).

[2] X. Liu, An overview of $X Y Z$ new particles, Chin. Sci. Bull. 59, 3815 (2014).

[3] F. K. Guo, C. Hanhart, U. G. Meiner, Q. Wang, Q. Zhao, and B. S. Zou, Hadronic molecules, Rev. Mod. Phys. 90, 015004 (2018).

[4] J. J. Wu, R. Molina, E. Oset, and B. S. Zou, Prediction of Narrow $N^{*}$ and $\Lambda^{*}$ Resonances with Hidden Charm above 4 GeV, Phys. Rev. Lett. 105, 232001 (2010).

[5] Z. C. Yang, Z. F. Sun, J. He, X. Liu, and S. L. Zhu, The possible hidden-charm molecular baryons composed of anti-charmed meson and charmed baryon, Chin. Phys. C 36, 6 (2012).
[6] W. L. Wang, F. Huang, Z. Y. Zhang, and B. S. Zou, $\Sigma_{c} \bar{D}$ and $\Lambda_{c} \bar{D}$ states in a chiral quark model, Phys. Rev. C 84, 015203 (2011).

[7] J. J. Wu, T.-S. H. Lee, and B. S. Zou, Nucleon resonances with hidden charm in coupled-channel models, Phys. Rev. C 85, 044002 (2012).

[8] X. Q. Li and X. Liu, A possible global group structure for exotic states, Eur. Phys. J. C 74, 3198 (2014).

[9] R. Aaij et al. (LHCb Collaboration), Observation of $J / \psi$ Resonances Consistent with Pentaquark States in $\Lambda_{b}^{0} \rightarrow J / \psi K^{-} p$ Decays, Phys. Rev. Lett. 115, 072001 (2015).

[10] R. Chen, X. Liu, X. Q. Li, and S. L. Zhu, Identifying Exotic Hidden-Charm Pentaquarks, Phys. Rev. Lett. 115, 132002 (2015). 
[11] H. X. Chen, W. Chen, X. Liu, T. G. Steele, and S. L. Zhu, Towards Exotic Hidden-Charm Pentaquarks in QCD, Phys. Rev. Lett. 115, 172001 (2015).

[12] M. Karliner and J. L. Rosner, New Exotic Meson and Baryon Resonances from Doubly-Heavy Hadronic Molecules, Phys. Rev. Lett. 115, 122001 (2015).

[13] L. Maiani, A. D. Polosa, and V. Riquer, The new pentaquarks in the diquark model, Phys. Lett. B 749, 289 (2015).

[14] V. V. Anisovich, M. A. Matveev, J. Nyiri, A. V. Sarantsev, and A. N. Semenova, Pentaquarks and resonances in the $p J / \psi$ spectrum, arXiv:1507.07652.

[15] R. F. Lebed, The pentaquark candidates in the dynamical diquark picture, Phys. Lett. B 749, 454 (2015).

[16] Z. G. Wang and T. Huang, Analysis of the $\frac{1}{2}^{ \pm}$pentaquark states in the diquark model with QCD sum rules, Eur. Phys. J. C 76, 43 (2016).

[17] Tomasz Skwarnicki on behalf of the LHCb Collaboration, Hadron spectroscopy and exotic states at LHCb, Proceedings at Moriond QCD 2019, http://moriond.in2p3.fr/2019/ QCD/Program.html.

[18] R. Aaij et al. (LHCb Collaboration), Observation of a narrow pentaquark state, $P_{c}(4312)^{+}$, and of two-peak structure of the $P_{c}(4450)^{+}$, Phys. Rev. Lett. 122, 222001 (2019).

[19] X. Liu, Y. R. Liu, W. Z. Deng, and S. L. Zhu, Is $Z^{+}(4430)$ a loosely bound molecular state?, Phys. Rev. D 77, 034003 (2008).

[20] X. Liu, Y. R. Liu, W. Z. Deng, and S. L. Zhu, $Z^{+}(4430)$ as a $D_{1}^{\prime} D^{*}\left(D_{1} D^{*}\right)$ molecular state, Phys. Rev. D 77, 094015 (2008).
[21] Y. R. Liu, X. Liu, W. Z. Deng, and S. L. Zhu, Is X(3872) really a molecular state?, Eur. Phys. J. C 56, 63 (2008).

[22] X. Liu, Z. G. Luo, Y. R. Liu, and S. L. Zhu, X(3872) and other possible heavy molecular states, Eur. Phys. J. C 61, 411 (2009).

[23] X. Liu and S. L. Zhu, $Y(4143)$ is probably a molecular partner of $Y(3930)$, Phys. Rev. D 80, 017502(E) (2009); Erratum, Phys. Rev. D 85, 019902(E) (2012).

[24] F. Close and C. Downum, On the Possibility of Deeply Bound Hadronic Molecules from Single Pion Exchange, Phys. Rev. Lett. 102, 242003 (2009).

[25] Y. R. Liu and M. Oka, $\Lambda_{c} N$ bound states revisited, Phys. Rev. D 85, 014015 (2012).

[26] T. M. Yan, H. Y. Cheng, C. Y. Cheung, G. L. Lin, Y. C. Lin, and H. L. Yu, Heavy quark symmetry and chiral dynamics, Phys. Rev. D 46, 1148(E) (1992); Erratum, Phys. Rev. D 55, 5851(E) (1997).

[27] M. B. Wise, Chiral perturbation theory for hadrons containing a heavy quark, Phys. Rev. D 45, R2188 (1992).

[28] G. Burdman and J. F. Donoghue, Union of chiral and heavy quark symmetries, Phys. Lett. B 280, 287 (1992).

[29] R. Casalbuoni, A. Deandrea, N. Di Bartolomeo, R. Gatto, F. Feruglio, and G. Nardulli, Phenomenology of heavy meson chiral Lagrangians, Phys. Rep. 281, 145 (1997).

[30] A. F. Falk and M. E. Luke, Strong decays of excited heavy mesons in chiral perturbation theory, Phys. Lett. B 292, 119 (1992).

[31] M. Tanabashi et al. (Particle Data Group), Review of particle physics, Phys. Rev. D 98, 030001 (2018). 